\title{
Educación sostenible y conservación del patrimonio cultural en la formación del profesorado universitario
}

\author{
María Pilar Molina-Torres ${ }^{1}$ y Raimundo Ortiz-Urbano ${ }^{2}$ \\ (1) Facultad de Ciencias de la Educación, Dpto. de Didácticas Específicas, Univ. de Córdoba, España \\ (Correo-e: pilar.molina@uco.es) \\ (2) Cabildo Catedral de Córdoba, Calle Cardenal Herrero 1, Córdoba, España \\ (Correo-e: raimundo_ortiz@hotmail.com)
}

Recibido Jun. 24, 2020; Aceptado Ago. 25, 2020; Versión final Sep. 21, 2020, Publicado Feb. 2021

\begin{abstract}
Resumen
El objetivo de este estudio es evaluar cómo se implementan las estrategias metodológicas empleadas para desarrollar competencias en sostenibilidad y un compromiso patrimonial en los estudiantes. La educación para el desarrollo sostenible se centra en la concienciación para la conservación de los bienes patrimoniales y naturales. La muestra la componen 458 alumnos y alumnas del grado de educación primaria de las Universidades de Cádiz y de Córdoba (España). Se ha optado por una metodología mixta con un diseño de corte transversal, fundamentalmente descriptivo e interpretativo, que se complementó con un diseño cuantitativo no experimental tipo encuesta. Los resultados arrojaron diversas creencias acerca de la afinidad que existe entre el patrimonio cultural y la educación sostenible a nivel curricular y metodológico. En conclusión, este proyecto supone una significativa oportunidad para que el alumnado universitario revise y supere la excesiva teorización de su actual formación universitaria.
\end{abstract}

Palabras clave: formación en competencias; educación ciudadana; enseñanza universitaria; patrimonio

\section{Sustainable education and conservation of cultural heritage for teacher education students}

\begin{abstract}
The objective of the present study is to evaluate how methodological strategies are implemented when developing student sustainability competencies and cultural heritage. Sustainable development education focuses on raising awareness about heritage and natural assets conservation. The sample is composed of 458 students from the primary education degree program at the Universities of Cádiz and Córdoba (Spain). A mixed transversal design methodology (descriptive and interpretative) is applied with a quantitative nonexperimental design (survey). The results show diverse beliefs about the affinity that exists between cultural heritage and sustainable education at the curricular and methodological levels. In conclusion, the present study opens an opportunity for university students to review and overcome excessive theorization in higher education.
\end{abstract}




\section{INTRODUCCIÓN}

La sostenibilidad cultural y la conservación del patrimonio son dos conceptos que se aúnan en una sociedad globalizada y al mismo tiempo localmente desigual. Las oportunidades y contratiempos que se atribuyen a este vínculo han tenido varias lecturas. En la actualidad, esa misma sociedad está inmersa en una serie de desafíos y retos que comportan numerosos avances en la economía, el medio ambiente y la cultura del desarrollo sostenible. Sin embargo, aunque la relación entre individuo y sociedad va cambiando progresivamente, el desarrollo humano sostenible actúa como un indicador vinculado principalmente al crecimiento económico, aunque también al social, cultural y medioambiental. Esta situación ha motivado que el estado actual de la investigación en las dos últimas décadas haya dado como resultados diferentes estudios (Barthel-Bouchier, 2013; Harrison, 2013; Junyent y Geli, 2008; Sterling y Thomas, 2006; Stubbs, 2004; Tweed y Sutherland, 2007). La tendencia actual es distinguir entre los términos cultura y sostenibilidad que se encuentran interrelacionados y sujetos a procesos políticos, sociales y científicos, y que, en cualquier caso, dificultan una definición final (Soini y Dessein, 2016).

Ahora bien, las propuestas curriculares actuales no incluyen la sostenibilidad para la formación en competencias del profesorado universitario. Es más, la Educación para la Sostenibilidad no es una realidad que pueda percibirse en las aulas universitarias. La universidad tiene la responsabilidad de formar a futuros docentes e integrar a la cultura sostenible en sus procesos formativos como un factor esencial para promover una educación para la sostenibilidad en el profesorado y también en la ciudadanía que éstos formarán (Albareda et al., 2019). Para conseguir estos cambios y poder implementarlos en el ámbito educativo es necesario la participación a nivel comunitario para con ello promover la sostenibilidad y la responsabilidad social. De hecho, son dos ideas claves, la ciudadanía global y el voluntariado, las que forman parte de la educación para la sostenibilidad.

Por su parte, Geli y Leal (2006) afirman que es necesario considerar la contextualización en el espacio local y global, y en el tiempo (históricamente, en el pasado, presente y con una visión del futuro) de manera integrada y holística. Desde la perspectiva de la educación patrimonial, Marín y Fontal (2020) apuntan que debe ser un proceso que forme parte de la educación de un individuo, tanto en el proceso de educación formal como informal. Es más cuando al hablar de historical thinking, el pensar históricamente no se ha centrado solamente en recordar acontecimientos pasados, sino en comprender e interpretar los hechos históricos y las razones que dependen de determinados contextos temporales y geográficos (Seixas y Morton, 2013). En este sentido, el ámbito de la educación ciudadana y cultural también ha alcanzado una posición destacada. La formación de la ciudadanía redunda en la educación en valores y el desarrollo humano sostenible. A este propósito los estudiantes aprenden e implementan en la práctica universitaria sus competencias profesionales a través de un contexto educativo real (Albareda y Alférez, 2016). De este modo, la concreción en el aula de los elementos curriculares motivará diferentes formas de pensar de los futuros profesionales de la educación patrimonial.

Para ello, uno de los retos universitarios es la incorporación de la sostenibilidad en el currículum de la educación superior como componente fundamental de la formación de los futuros profesionales. Es necesario que la formación inicial deba mejorar desde sus niveles básicos para orientarla al ejercicio profesional y su acceso a la función docente (O’Byrne et al., 2015). Al respecto Jiménez et al., (2018) apuntan que la formación didáctica del profesorado en los métodos de enseñanza es una oportunidad para el aprendizaje del docente y una mayor implementación de éstos en su desarrollo profesional. También hay que tener en cuenta que este enfoque metodológico debe venir acompañado de estrategias que favorezcan la búsqueda de soluciones a los problemas que se vivencian en el mundo real. Es a raíz de esta investigación de cariz social que se suscita la búsqueda de respuestas y se fomentan actitudes que promueven competencias ciudadanas (Molina-Torres, 2019). Desde esta perspectiva, la sostenibilidad debe entenderse como el equilibrio y el mantenimiento a largo plazo de los recursos y procesos naturales y culturales en un territorio concreto (Feria, 2012). De ahí que la adquisición de las competencias ciudadanas en el ámbito universitario permita la conservación del entorno y el sentido de pertenencia a la cultura ambiental (De La Cruz, 2018). Así la educación para la sostenibilidad supone un triple enfoque de educación ambiental, educación en ciencias sociales y educación ciudadana.

Por otra parte, son varios los obstáculos que tienen los docentes y que marcan su escasa cultura de sostenibilidad patrimonial en la universidad. Entre éstos destacan la implementación de metodologías más tradicionales que saturan conceptualmente el currículo en los estudios de Grado. La formación del alumnado del Grado de Educación Primaria debe encaminarse en la búsqueda de habilidades, destrezas y conocimientos que garanticen en sus aplicaciones prácticas un progreso de las estrategias metodológicas. Se trata, en definitiva, de acometer un cambio en la metodología de enseñanza que centre el objetivo en el proceso de aprendizaje del estudiante, en un contexto educativo que se extiende a lo largo de la vida académica. Así, a la hora de ejecutar la presente propuesta de investigación, se han tenido en cuenta los modelos didácticos que el 
alumnado universitario de Educación Primaria elegiría para sus clases y la autonomía que adquieren durante su formación, para dar prioridad a las competencias educativas sobre los contenidos teóricos (tabla 1).

Tabla 1: Metodologías docentes elegidas por el alumnado para educar en sostenibilidad y patrimonio. Fuente: elaboración propia.

\begin{tabular}{|l|}
\hline -Aprendizaje servicio \\
-Aprendizaje cooperativo \\
-Aprendizaje Basado en Proyectos (ABP) \\
-Flipped Classroom \\
-Gamificación \\
-Aprendizaje Basado en Competencias \\
\hline
\end{tabular}

En este punto, la educación patrimonial no persigue formar expertos en un tema, sino despertar la curiosidad y la sensación de pertenencia a un lugar, a partir del conocimiento de sus referentes más próximos, para poder hacer uso de ellos de forma sostenible (Barth et al., 2007). Esta mirada permite comprender y explicar el valor de los elementos patrimoniales como referentes de la memoria de la comunidad en una dimensión simbólico-identitaria, superando la consideración material respecto a la dimensión histórico-artística. Por todo ello, el objetivo prioritario de esta investigación ha sido la recopilación de estrategias metodológicas empleadas para desarrollar competencias en sostenibilidad y compromiso patrimonial. Para alcanzar este objetivo principal también se recogen los siguientes objetivos específicos: (1) conocer metodologías activas de aprendizaje; (2) combinar la investigación y la enseñanza de las ciencias sociales y la educación patrimonial; (3) analizar las dificultades académicas de los estudiantes para reconocer los contenidos de sostenibilidad cultural y patrimonial en su formación, y, por último, (4) crear prácticas sostenibles y patrimoniales en su enseñanza universitaria.

\section{METODOLOGÍA}

El método que hemos implementado se asemeja a las características de una metodología de indagación. Las particularidades investigativas de la experiencia detallan la formación metodológica del profesorado del Grado de Educación Primaria. Ahora bien, centrándonos en la temática a tratar el estudio describe un problema de investigación que se caracteriza por la escasez de investigaciones relacionadas con la gestión sostenible del patrimonio cultural. Sin embargo, el enfoque metodológico de este estudio tiene como precedentes el enriquecedor trabajo de Albareda et al., (2018), en cuanto al diseño y la metodología, con el fin de explorar los elementos vinculados al desarrollo sostenible y las prácticas de enseñanza-aprendizaje en sostenibilidad.

\section{Participantes y muestra}

La elección de la población de estudio ( $\mathrm{N}=458$ ) está constituida por el alumnado del Grado de Educación Primaria del $2^{\circ}$ ciclo. El criterio para tal selección se hizo con la intención de comparar y contextualizar la enseñanza de dos asignaturas con contenidos semejantes desde el punto de vista didáctico, pero no conceptual, además de ser muestras de diferentes universidades. En referencia con la distribución, dos grupos cursaban la asignatura "Didáctica del Medio Ambiente" de cuarto año $(\mathrm{N}=123 ; 26,8 \%)$ en la Universidad de Córdoba. Mientras que cinco grupos cursaban la asignatura "Didáctica de las Ciencias Sociales", de tercer curso ( $N=335 ; 73,2 \%)$, concretamente 134 alumnos en la Universidad de Cádiz y 201 en la de Córdoba. La edad media de los estudiantes que participaron en esta investigación es de 23 años.

Para la selección de la muestra que no tuvo un carácter probabilístico, tuvimos en cuenta la muestra total del estudio en la que las dos docentes responsables de las asignaturas se habían implicado de forma directa en la investigación educativa. En ambas universidades se implementó la experiencia investigativa siguiendo una línea de trabajo basada en el contraste profesionalizante de las competencias educativas de las dos disciplinas. Otro de los aspectos que destaca en este estudio son las diferencias competenciales y curriculares que tienen ambas materias (tabla 2). También es necesario señalar que, aunque el número de estudiantes de la asignatura de Didáctica del Medio Ambiente es inferior, la representatividad de la muestra es semejante.

\section{Diseño de la investigación}

Se utilizó un diseño de corte transversal, fundamentalmente descriptivo e interpretativo, propio de la investigación cualitativa que aplica la recogida y análisis de datos no numéricos (Kormblit, 2004), y que además se complementó con un diseño cuantitativo no experimental tipo encuesta. La línea de investigación donde se incluye el estudio se centró en analizar las metodologías activas de aprendizaje en la Didáctica de las Ciencias Sociales y la Didáctica del Medio Ambiente. En este sentido, para analizar la capacitación 
metodológica que el alumnado adquiere en ambas materias del Grado de Educación Primaria, se elaboraron varias cuestiones de carácter mixto siguiendo la propuesta de Kvale (2011). Asimismo, esta investigación daría respuesta a la evaluación de las diferentes competencias profesionales y los aprendizajes adquiridos en el campo de la investigación.

Tabla 2: Competencias profesionales para la formación universitaria de las siguientes asignaturas. Fuente: elaboración propia.

\begin{tabular}{|l|l|}
\hline Didáctica de las Ciencias Sociales & Didáctica del Medio Ambiente \\
\hline - Desarrollar y evaluar contenidos del currículum & - Integrar el estudio histórico y geográfico \\
mediante recursos didácticos apropiados y & desde una orientación instructiva y cultural. \\
promover las competencias correspondientes en & - Valorar la responsabilidad individual y \\
los estudiantes. & colectiva en la consecución de un futuro \\
- Asumir que el ejercicio de la función docente ha & sostenible. \\
de ir perfeccionándose y adaptándose a los & - Construir una visión actualizada del mundo \\
cambios científicos, pedagógicos y sociales a lo & natural y social. \\
largo de la vida. & - Fomentar la educación democrática de la \\
- Conocer modelos de mejora de la calidad con & ciudadanía y la práctica del pensamiento \\
aplicación a los centros educativos. & social crítico. \\
\hline
\end{tabular}

\section{Instrumento para la recogida de datos}

La toma de datos se realizó a través de la observación directa del alumnado y una encuesta con el fin de que reflexionaran sobre su formación inicial en la cultura sostenible y los métodos de enseñanza activos. Este tipo de estudios mediante encuesta son muy habituales en las investigaciones educativas, ya que pueden aplicarse a múltiples problemas y nos permiten asegurar la validez de la muestra (Sapsford y Jupp, 2006). La intervención se realizó en un total de tres sesiones prácticas de hora y media distribuidas durante tres semanas de las diez que dura el cuatrimestre. En las tres prácticas se involucraron la totalidad de la muestra $(\mathrm{N}=458)$, y fueron divididos en pequeños grupos de cuatro o cinco alumnos y alumnas.

Por otra parte, con el fin de dar respuesta a los objetivos planteados en la investigación, se analizó la información de los instrumentos a través de una recopilación de las narrativas más destacadas en sus diarios (Chárriez, 2012). El sistema de observación se materializó mediante un diario de clase de los pequeños grupos de estudiantes que sirvió como instrumento narrativo para reflejar sus valoraciones, hipótesis y conclusiones. Para ello, se tomó como referencia una encuesta de opinión de carácter cualitativo que recoge las opiniones del alumnado de ambas asignaturas con respecto a varias categorías relacionadas con la sostenibilidad, el entorno urbano y/o rural, la empatía y el patrimonio histórico (tabla 3).

Tabla 3: Categorías sobre términos sostenibles y patrimoniales. Fuente: elaboración propia.

\begin{tabular}{|l|l|}
\hline Categorías & Cuestiones \\
\hline Relación con la sostenibilidad cultural & $\begin{array}{l}\text { Correspondencia entre cultura y desarrollo } \\
\text { sostenibles }\end{array}$ \\
\hline Gestión de la educación ambiental & $\begin{array}{l}\text { Cuidado responsable de los espacios } \\
\text { naturales de nuestra ciudad }\end{array}$ \\
\hline Descripción de un entorno urbano y/o rural & $\begin{array}{l}\text { Tipologías de un contexto urbanizado y/o } \\
\text { ruralizado }\end{array}$ \\
\hline $\begin{array}{l}\text { Conservación y preservación del } \\
\text { patrimonio }\end{array}$ & $\begin{array}{l}\text { Importancia histórica de las estructuras y } \\
\text { monumentos de nuestra ciudad }\end{array}$ \\
\hline $\begin{array}{l}\text { Narrativas históricas y empatía con el } \\
\text { espacio habitado y estudiado }\end{array}$ & $\begin{array}{l}\text { Emplazamiento geográfico e histórico de } \\
\text { los bienes culturales del pasado }\end{array}$ \\
\hline
\end{tabular}

Para el diseño y validación de la encuesta que se denominó "Creencias para la sostenibilidad cultural y el patrimonio histórico en metodologías activas de aprendizaje", intervinieron cinco expertos en educación sostenible y patrimonial, y en metodologías de enseñanza activa de universidades españolas. De la valoración de las diferentes categorías y cuestiones (tabla 3), y de los valores y pertinencia de los ítems de la tabla 4, se obtuvo el resultado final de ambas herramientas. Se consideraron todas las recomendaciones y tras la aprobación de su estructura y contenidos, se configuró la tabla 3 con varias cuestiones agrupadas en categorías para que los participantes de ambas universidades expresaran abiertamente sus ideas, y otra con una panorámica general de los métodos de enseñanza que articulan su docencia universitaria (tabla 4). Esta última fue validada con una escala tipo Likert de cinco valores que oscilan del 1 (totalmente en desacuerdo) al 5 (totalmente de acuerdo). 
Tabla 4: Cuestionario sobre los métodos de aprendizaje más utilizados en las asignaturas del estudio. Fuente: elaboración propia.

\begin{tabular}{|l|l|l|l|l|l|}
\hline Valoración & 1 & 2 & 3 & 4 & 5 \\
\hline El docente explicaba de manera transmisiva & & & & & \\
\hline $\begin{array}{l}\text { La enseñanza expositiva se basa en la } \\
\text { memorización de conceptos }\end{array}$ & & & & & \\
\hline El profesorado organiza seminarios prácticos & & & & & \\
\hline $\begin{array}{l}\text { El docente establece prácticas en contextos } \\
\text { educativos }\end{array}$ & & & & & \\
\hline $\begin{array}{l}\text { El docente facilita los trabajos de indagación e } \\
\text { investigación }\end{array}$ & & & & & \\
\hline $\begin{array}{l}\text { Las sesiones tutoriales refuerzan los métodos de } \\
\text { enseñanza }\end{array}$ & & & & & \\
\hline $\begin{array}{l}\text { El trabajo autónomo y cooperativo consolida el } \\
\text { aprendizaje metodológico }\end{array}$ & & & & & \\
\hline El docente utiliza materiales para diseñar proyectos & & & & & \\
\hline Uso de talleres prácticos por parte del docente & & & & & \\
\hline El docente propone la resolución de tareas & & & & & \\
\hline $\begin{array}{l}\text { El docente plantea estudios de caso que fomentan } \\
\text { el trabajo por competencias }\end{array}$ & & & & & \\
\hline $\begin{array}{l}\text { El método investigativo se basa en la búsqueda de } \\
\text { información metodológica }\end{array}$ & & & & & \\
\hline $\begin{array}{l}\text { Los trabajos de síntesis facilitan el conocimiento de } \\
\text { diferentes métodos }\end{array}$ & & & & & \\
\hline
\end{tabular}

Este instrumento de investigación complementa la evaluación cualitativa del estudio. Para ello, sus contribuciones se centran en las percepciones de los participantes para detectar cuáles son los métodos de enseñanza tradicionales o activos/participativos más adecuados y productivos, para trabajar en el aula de Educación Primaria la sostenibilidad cultural y la educación patrimonial.

\section{RESULTADOS}

Los resultados de la evaluación cualitativa que se muestran a continuación reflejan las dificultades del alumnado para trasladar los conceptos a la práctica y construirlos a partir de la reflexión (tabla 3). Las carencias conceptuales proporcionan una descripción precisa de su falta de conocimientos para su desarrollo profesional. De todas las aportaciones del alumnado es curioso cómo no son capaces de relacionar sostenibilidad con Educación ambiental y los bienes patrimoniales e históricos de su ciudad. En sus apreciaciones inciden en la valoración del patrimonio natural y cultural, aunque no tengan claro la relación de los tópicos y de las categorías que se les presentaban para comentarlas. Las temáticas más destacadas se centraron en las metodologías recibidas en las sesiones teórico-prácticas de ambas disciplinas: Didáctica de

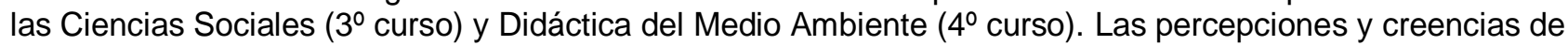
las categorías investigadas se recogieron de manera generalista, aunque pertenezcan a diferentes cursos y asignaturas. Las opiniones se centraron en sus experiencias de aprendizaje personal. Las respuestas a cada una de las cuestiones describen con precisión la relación entre cultura histórica y desarrollo sostenibles.

En este sentido, los estudiantes afirmaban en cuanto a los métodos de enseñanza recibidos en la universidad: "los métodos utilizados por el profesorado no son útiles para nuestra formación como futuros docentes" (E303, E211, E048). Estas aportaciones muestran la repercusión y la implementación de un método de aprendizaje tradicional en una materia como la educación sostenible y la cultura patrimonial en la que se utilizan recursos educativos poco novedosos como el copiado de apuntes y las pruebas teóricas, lo que hace que se continúe primando el relato del docente. Al hilo se afirma: "Las sesiones teóricas y prácticas son repetitivas y se centran en memorizar conceptos y la explicación del profesor" (E089, E135). Es más que evidente que la formación de los alumnos no refleja un procedimiento centrado en la experimentación con problemas prácticos basados en situaciones reales, sino más bien un enfoque principalmente teórico. Por este motivo, el alumnado demanda: "Nos gustaría conocer en que consiste el aprendizaje basado en proyectos y otras metodologías más activas" (E058, E093, E348). Es una realidad que la oportunidad de adquirir conocimientos metodológicos más innovadores redunda en el desarrollo de las competencias profesionales desde la universidad. Asimismo, Lambrechts y Van Petegem (2016) puntualizan que estas competencias tienen un fuerte enfoque en la complejidad, la interconexión, las actitudes y los valores, por lo que es particularmente difícil tratarlas en el contexto educativo actual basado en competencias. 
En cuando a la formación por competencias educativas afirmaban: "El trabajo por competencias no se recoge en nuestras sesiones prácticas en la universidad" (E052, E076, E198). Y añaden: "Los contenidos explicados por el docente no son motivadores" (E099, E033, E226). Del mismo modo, cuando relatan sus experiencias con los métodos de aprendizaje más activos señalan: "Las metodologías activas de aprendizaje no son implementadas en ambas asignaturas" (E372, E013, E062, E167). En este sentido, nos dicen: "Conocemos algunos instrumentos y recursos didácticos más innovadores por otras materias" (E046, E257), a lo que añaden "Ios profesores no utilizan el patrimonio natural ni tampoco cultural para conocer la gestión sostenible del entorno" (E234, E348, E049, E068). Lo que indicaría una falta de interés del profesorado para incluir en el sistema educativo ejemplos de sostenibilidad curricular en la universidad. Por último, resaltan: "El uso de las TIC debe ir encaminado a la búsqueda y el análisis de los recursos históricos y sostenibles" (E450, E309); y también: "Las herramientas digitales proporcionan nuevos conocimientos actualizados para detectar las problemáticas económicas, sociales y ambientales" (E018, E176, E071). Sus afirmaciones muestran que están de acuerdo en realizar investigaciones y en utilizar recursos digitales para interpretar los bienes patrimoniales y naturales.

Al hilo de las cuestiones planteadas con relación al patrimonio y la empatía históricas enfatizan unos resultados y unas conclusiones experimentales. Las opiniones comparten un factor común: el acercamiento que tienen hacia los bienes patrimoniales que no asocian con la sostenibilidad cultural. De hecho, esta influencia directa del patrimonio cultural en la enseñanza supone un reto para identificar la importancia que tiene la educación patrimonial en el contexto curricular universitario y la conciencia histórica para conformar la identidad patrimonial del alumnado. Sin embargo, una de esas dificultades que obstaculizan la formación del alumnado universitario se debe al desconocimiento de los estudiantes en lo que respecta a su entorno patrimonial. De este modo, indicaban: "Pienso que un monumento como la Mezquita se situó aquí geográficamente por su importancia histórica" (E355, E034, E188), sin embargo, "las causas y consecuencias no las sé, pero podrían ser por la importancia histórica de nuestra ciudad" (E051, E106, E273). Puntualizan también: "Conozco mejor el entorno del casco antiguo por una salida didáctica con mi grupo de clase en la universidad" (E037, E282).

Es obvio que el mantenimiento de una metodología tradicional no ha conseguido formar personas culturalmente más sostenibles. En la práctica, las opiniones del alumnado centraban su interés en expresar las dudas que tenían sobre el desarrollo sostenible y cultural: "Desconozco la relación y que significa el desarrollo sostenible y, por otro lado, la cultura (sostenible)" (E405); "Creo que desarrollo y cultura sostenibles no están relacionados" (E266). Sin embargo, es evidente que los conocimientos sobre educación ambiental no son tan desconocidos. Piensan en una gestión responsable de los espacios naturales de nuestra ciudad, como la conservación del entorno natural del río Guadalquivir. En esta dirección, se preguntaron: "¿es un espacio natural protegido?, ¿la legislación preserva estos monumentos naturales?, ¿qué instituciones los conservan?," (E065, E093, E109). Por otra parte, las interpretaciones acerca de un lugar que estuviese en un emplazamiento urbano o rural de la ciudad fueron muy concretos. Destacaban: "Para mí la diferencia entre un entorno urbano y uno rural es que el primero está habitado por el ser humano y el segundo no" (E440, E010). A lo que añadían discretamente: "creo que urbano sería una ciudad y rural un campo o un terreno en el que no vive nadie" (E088). De estas afirmaciones, podemos percibir que no saben diferenciar un espacio natural de uno más urbanizado, ni tampoco describir un paisaje urbano o un espacio rural.

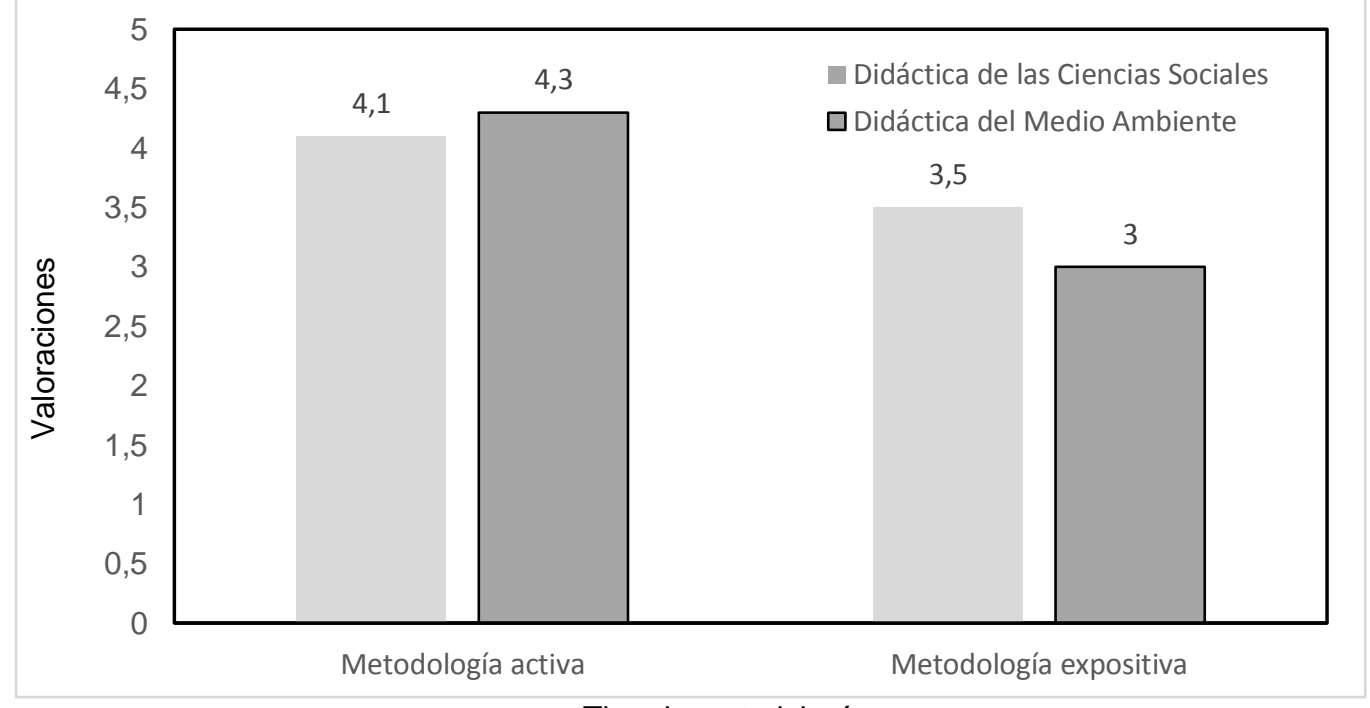

Tipo de metodología

Fig. 1: Valoraciones metodológicas del alumnado para ambas materias. Fuente: elaboración propia. 
Analizadas las respuestas de ambas asignaturas, la figura 1 muestra una comparativa de las valoraciones de la tabla 4. Encontramos con un porcentaje de 3,5 y 3 la metodología expositiva, mientras que entre el 4,1 y el 4,3 de las respuestas confirman que una metodología participativa favorece un aprendizaje activo y significativo. Así pues, utilizando esta metodología mixta, se identificaron diferentes visiones, dificultades y desafíos en relación con los contenidos y estrategias de la enseñanza (Burke y Christensen, 2014) y la educación para la sostenibilidad. Abordar esos conceptos permitió describir y detectar las valoraciones del alumnado y los diferentes tipos de metodologías de enseñanza adquiridas en sus estudios de grado (Wright, 2004). No obstante, dejan claro con sus reflexiones que el trabajo autónomo y la retroalimentación en grupos colaborativos ocupa un lugar destacado en su formación universitaria, aunque reconocen que se han esforzado más para relacionar la teoría y la práctica mediante la memorización de los conceptos teóricos, lo que no enriquece su futuro profesional docente.

\section{DISCUSIÓN FINAL}

En líneas generales, las creencias del alumnado de ambas universidades son un elemento determinante para averiguar sus carencias metodológicas y el desconocimiento de los contenidos de carácter sostenible y patrimonial. Este trasfondo tiene una repercusión negativa que les hace acostumbrarse a trabajar de manera tradicional para aprender las problemáticas de la sostenibilidad cultural. El reto para pensar histórica y sosteniblemente reside en la formación de los futuros docentes en el ámbito universitario, en etapas educativas como la de Educación Primaria. Esta formación universitaria no ahonda en los aspectos más relevantes para su futuro desempeño docente ni tampoco trabaja las competencias necesarias para abordar problemas de aula reales (Gairín, 2011). De este modo, es necesario tener en cuenta que este enfoque metodológico debe venir acompañado de estrategias que favorezcan la búsqueda de soluciones a los problemas que se vivencian en el mundo real, y no se ciñan a la memorización de contenidos conceptuales que tienen poco sentido en una sociedad donde la información está disponible fácilmente.

Por su parte, Albareda et al., (2019) elaboraron una rúbrica para analizar las competencias genéricas en sostenibilidad y constatar que el nivel de introducción de la sostenibilidad en las actividades de enseñanzaaprendizaje es relativamente limitado. Entre las expectativas de esta propuesta se discuten los distintos niveles de logro de competencias, teniendo en cuenta, por un lado, los intereses del alumnado en su proceso formativo, y, por otro lado, que todas las materias no tienen el mismo nivel de adquisición de competencias en sostenibilidad. Esta experiencia también fue desarrollada con estudiantes y docentes de Educación Primaria que reconocen la importancia de las competencias ciudadanas en la práctica de los centros docentes y de la comunidad en general (De La Cruz, 2018). Sin embargo, pese a la formación en competencias ciudadanas y sostenibles y el uso de éstas en los centros educativos, la enseñanza para la sostenibilidad cultural continúa adscrita al modelo expositivo de aprendizaje y a la prueba escrita para evaluar contenidos conceptuales. Estas prácticas no permiten analizar los acontecimientos del presente y el pasado y establecer diferencias o semejanzas que hagan del patrimonio cultural y el desarrollo sostenible una disciplina interesante.

Recordemos que las narrativas personales del alumnado inciden en la insuficiente preparación de los profesores noveles y los problemas que tienen para diferenciar conceptos como sostenibilidad y educación ambiental. Al respecto Bonil et al., (2010) afirman que la educación ambiental se configura como un espacio de encuentro permanente entre alumnado, docentes y saberes, orientado hacia la finalidad común concretada en una forma de entender la formación ciudadana. Ahora bien, las concepciones de los futuros docentes de Educación Primaria tienen un aspecto positivo y en común, sus respuestas afirmativas en las cuestiones relacionadas con la educación sostenible y el impacto práctico que tiene en su formación profesional. Pese a las opiniones relacionadas con el aprendizaje de estas temáticas para facilitar su conocimiento e implementación en un aula, se plantean dudas sobre la función y definición de estos bienes. Como indican O’Byrne et al., (2015), a pesar de la proliferación del trabajo académico para proponer definiciones y estándares para el campo de la sostenibilidad y sus conceptos centrales, se ha trabajado menos para evaluar el contenido estatal y curricular de los programas de grado existentes en sostenibilidad.

También, pese a los resultados de este estudio, la educación para la sostenibilidad del alumnado aporta ciertas dudas acerca de las prácticas educativas que pueden reproducir en sus clases de Educación Primaria. Es aquí donde las propuestas de mejora en su formación personal les hacen implicarse y encauzar sus prácticas profesionales (Imbernón, 2020). En esta dirección, una adecuada formación inicial del profesorado se traduce en la adquisición de elementos didácticos fundamentales para el proceso de enseñanza-aprendizaje que, a su vez, les permitan diseñar actuaciones específicas para abordar las causas y las consecuencias de una educación para la sostenibilidad. En nuestra investigación además de cumplir los objetivos planteados, hemos concluido que las dificultades del alumnado para afrontar una metodología innovadora y más activa depende no solamente de la formación del docente universitario, sino de la prioridad que se da a los contenidos conceptuales frente a las competencias educativas. 


\section{CONCLUSIONES}

De acuerdo al trabajo presentado y a los resultados obtenidos, se pueden plantear las siguientes conclusiones principales:

1.- Desde una perspectiva curricular, la sostenibilidad ambiental y la conservación del patrimonio en el contexto universitario no está totalmente implantada en los planes de estudios.

2.- Los métodos de enseñanza implementados en las dos materias analizadas, Didáctica de las Ciencias Sociales y Didáctica del Medio Ambiente, no son metodologías activas de aprendizaje, lo que refleja que los métodos transmisivos y conceptuales del aprendizaje en educación sostenible y patrimonial no enriquecen el aprendizaje de estas disciplinas.

3.- La formación del profesorado universitario no es la adecuada para desempeñar su trayectoria profesional por competencias en un contexto educativo e intercambiar propuestas y reflexionar sobre los métodos didácticos que les enriquezcan en sus prácticas como docentes.

4.- Las creencias y las percepciones de los estudiantes son realistas con respecto al aprendizaje que adquieren en sus grados universitarios, y perciben que su formación en sostenibilidad cultural y la educación patrimonial es mejorable para utilizarla en su futuro profesional.

\section{AGRADECIMIENTOS}

Este trabajo forma parte del proyecto de investigación HAR2015-68059-C2-1-R, financiado por el Ministerio de Economía y Competitividad del Gobierno de España.

\section{REFERENCIAS}

Albareda, S. y Alférez, A., A collaborative programme in sustainability and social responsibility, doi: 10.1108/IJSHE-072016-0134, International Journal of Sustainability in Higher Education, 17(5), 719-736 (2016)

Albareda, S., Vidal, S. y Fernández, M., Implementing the sustainable development goals at University level, doi: 10.1108/IJSHE-05-2017-0069, International Journal of Sustainability in Higher Education, 19(3), 473-497 (2018)

Albareda, S., Azcárate, P., Muñoz, J. M., Valderrama, R. y otro autor., Evaluar competencias en sostenibilidad en los grados y posgrados de educación: propuesta de un instrumento, doi: 10.5565/rev/ensciencias.2670, Enseñanza de las ciencias: revista de investigación y experiencias didácticas, 37(3), 11-29 (2019)

Barth, M., Godemann, J., Rieckmann, M. y Stoltenberg, U., Developing key competencies for sustainable development in higher education, doi:10.1108/14676370710823582, International Journal of Sustainability in Higher Education, 8(4), 416430 (2007)

Barthel-Bouchier, D., Cultural Heritage and the Challenge of Sustainability, Walnut Creek, Left Coast Press (2013)

Bonil, J., Junyent, M. y Pujol, R. M., Educación para la Sostenibilidad desde la perspectiva de la complejidad, doi: 10.25267/Rev_Eureka_ensen_divulg_cienc.2010.v7.iextra.05, Revista Eureka sobre Enseñanza y Divulgación de las Ciencias, 7, 198-215 (2010)

Burke, R. y Christensen, L., Educational Research: Quantitative, Qualitative, and Mixed Approaches, California, Sage (2014)

Chárriez, M., Historias de vida: Una metodología de investigación cualitativa, Revista Griot, ISSN: 1949-4742, 5, 50-67 (2012)

De La Cruz, W., Las competencias ciudadanas como generadoras de cultura ambiental, doi: 10.17981/cultedusoc.9.2.2018.06, Cultura. Educación y Sociedad, 9(2), 67-76 (2018)

Feria, J. M. (Ed.), Territorial Heritage and Development, London, Taylor and Francis (2012)

Gairín, J., Formación de profesores basada en competencias, Bordón. Revista de Pedagogía, ISSN: 2340-6577, 63(1), 93-108 (2011)

Geli, A. M. y Leal, W., Education for sustainability in university studies: Experiences from a project involving European and Latin American universities, doi: 10.1108/14676370610639263, International Journal of Sustainability in Higher Education, 7(1), 81-93 (2006)

Harrison, R., Heritage: critical approaches, London, Routledge (2013)

Imbernón, F., En educación, no todo vale. Evidencias científicas para mejorar la práctica docente. Dossier Graó, ISSN: 2462-5949, 5, $111(2020)$

Jiménez, D., González, J. J. y Tornel, M., Formación del profesorado universitario, doi: 10.4067/S071807052018000300157, Estudios Pedagógicos, 44(3), 157-172 (2018) 
Junyent, M. y Geli, A. M., Education for sustainability in university studies: a model for reorienting the curriculum. British Educational Research Journal, doi: 10.1080/01411920802041343, 34(6), 762-783 (2008)

Kormblit, A. L. (Coord.)., Metodologías cualitativas en ciencias sociales, Buenos Aires, Editorial Bilblo (2004)

Kvale, S., Las entrevistas en investigación cualitativa, Madrid, Morata (2011)

Lambrechts, W. y Van Petegem, P., The interrelations between competences for sustainable development and research competences, doi: 10.1108/IJSHE-03-2015-0060, International Journal of Sustainability in Higher Education, 17, 776-795 (2016)

Marín, S. y Fontal, O., Percepciones de estudiantes de Educación Secundaria Obligatoria en torno al Patrimonio, doi:10.5209/aris.64657, Revista Arte, Individuo y Sociedad, 32(4), 917-933 (2020)

Molina-Torres, M. P., El Aprendizaje Basado en Proyectos (ABP) en la formación metodológica del profesorado del Grado de Educación Primaria, doi: 10.14201/et2019371123137, Enseñanza \& Teaching: Revista interuniversitaria de didáctica, 37(1), 123-137 (2019)

O'Byrne, D., Dripps, W. y Nicholas, K. A., Teaching and learning sustainability: An assessment of the curriculum content and structure of sustainability degree programs in higher education, doi: 10.1007/s11625-014-0251-y, Sustainability Science, 10(1), 43-59 (2015)

Sapsford, R. y Jupp, V., Data collection and analysis, London, Sage (2006)

Seixas, P. y Morton, T., The big six historical thinking concepts, Toronto, Nelson (2013)

Soini, K. y Dessein, J., Culture-Sustainability Relation: Towards a Conceptual Framework, doi: 10.3390/su8020167, Sustainability, 8(2), 167 (2016)

Sterling, S. y Thomas, I., Education for sustainability: the role of capabilities in guiding university curricula, doi: 10.1504/IJISD.2006.013735, International Journal of Innovation and Sustainable Development, 1(4), $349-370$ (2006)

Stubbs, M., Heritage-sustainability: developing a methodology for the sustainable appraisal of the historic environment, doi: 10.1080/0269745042000323229, Planning Practice \& Research, 19(3), 285-305 (2004)

Tweed, C. y Sutherland, M., Built cultural heritage and sustainable urban development, doi: 10.1016/j.landurbplan.2007.05.008, Landscape and Urban Planning, 83(1), 62-69 (2007)

Wright, T.S.A., Definitions and frameworks for environmental sustainability in higher education, doi: 10.1016/S09528733(02)00002-8, International Journal of Sustainability in Higher Education, 3(3), 203-220 (2004) 
